\title{
Designing Vehicle Form Based on Subjective Customer Impressions
}

\author{
Koichiro Yazaki, Hiroki Takimoto, Kakuro Amasaka \\ Aoyama Gakuin University, Kanagawa-ken, Japan
}

\begin{abstract}
Automotive body styling is determined by two key design elements: profile and form. In recent years, the authors have been able to quantify profile designs that embody consumer preferences and verify the effectiveness of these efforts. This study focuses on form, quantifying parameters like roundness and angularity in order to identify the relationship of these qualities to customer preferences. In order to fully understand the relationship between form as a whole (which consists of front, side, and rear elements) and consumer preference, 3D-CAD software and statistics are used to quantify form. An eye-tracking camera is then used to analyze line of sight and establish a cause-and-effect relationship between form and where customers focus their attention, and the desired insights are obtained.
\end{abstract}

Keywords: automotive, designing vehicle form, 3D-CAD

\section{Introduction}

In developing a new product design, it is absolutely essential that designers have an accurate understanding of consumer preferences and values and reflect this information in the new product. Doing this requires that they take a scientific approach to customer preferences and apply a method that supports the generation of design ideas. They must put words to (or develop images and concepts for) unspoken customer feelings and incorporate these in their drawings through an explicit designing process (Amasaka, Nagaya, \& Shibata, 1999; Amasaka, 2007; Yamaji \& Amasaka, 2009).

This study presents applied research on a method to support the generation of automotive design ideas with a focus on vehicle form. Automotive body styling is determined by two key elements: profile (sense of proportion when seen from a distance) and form (surface undulations when seen up close). In recent years, the authors have been able to quantify (make proportional ratios explicit in) profile designs that embody (unspoken) consumer preferences and verify the effectiveness of these efforts.

We focus on form, quantifying parameters like roundness and angularity in order to identify the relationship of these qualities to customer preferences. Specifically, in order to fully understand the relationship between form as a whole (which consists of front, side, and rear elements), the authors use 3D-CAD software and statistics to quantify form, and then an eye-tracking camera to analyze line of sight and establish a

Koichiro Yazaki, Graduate Student, School of Science and Engineering, Aoyama Gakuin University. Hiroki Takimoto, Graduate Student, School of Science and Engineering, Aoyama Gakuin University.

Kakuro Amasaka, Ph.D., Professor, School of Science and Engineering, Aoyama Gakuin University.

Correspondence concerning this article should be addressed to Kakuro Amasaka, 5-10-1, Fuchinobe, Chuo-ku, Sagamihara-shi, Kanagawa-ken, 252-5258, Japan. E-mail: kakuro_amasaka@ise.aoyama.ac.jp. 
cause-and-effect relationship between form and where customers focus their attention. The desired results are obtained in the field of automotive design development.

\section{Background}

In order to develop and offer appealing and customer oriented products, it is important to incorporate customer feelings and words into the new product development process, and to fully put into practice the concept of "customer science". These days, changes in customer preferences towards automobiles are very pronounced, and are becoming increasingly diversified and complicated. In order to build functionally into durable goods, it is becoming important to use "sensitive engineering" that both quantifies and grasps the images and feelings in customers' hearts, such as their favorite foods.

Regarding automobile design, it is very important to use statistical methods to develop a commodity which precisely answers customer demands (Aoyama, 2004; Li, Kamaike, \& Ueda, 2007; Masuyama, 1996; Shinohara, Sakamoto, \& Shimizu, 1996; Yanagisawa \& Fukuda, 2004). Accordingly, various research studies have adopted this approach using "design SQC" and the "profile design support tool” (Amasaka, Nagaya, \& Shibata, 1999; Amasaka, 2005). Generally, customers notice three elements of the design: (1) profile design (proportions), which is noticed as long to mid-distance; (2) form, which is noticed in mid- to short-distance; and (3) surface, which is noticed at short-distance to close range (Asami, Ando, Yamaji, \& Amasaka, 2010).

This study focuses on form, quantifying parameters like roundness and angularity in order to identify the relationship of these qualities to customer preferences.

\section{Necessity of Numerical Form Representation by Computer Aided Design (CAD)}

In order to visualize the degree of influence of customer sensitivity due to the change of the form, digitalization of the form is indispensable. In this research, the CAD software is utilized as an expedient for form digitalization. The advantages of using CAD include: (1) "form digitalization" in which a numerical definition of the form is created is possible using CAD; (2) since the front, side, rear, etc. of the automobile are verifiable from all angles, establishing a relationship with customer sensitivity is possible; and (3) it may be noted that, as in the present design process, the use of CAD as a tool for expressing form for modeling etc. is mainstream use. Further, in this research, CATIA V5 is used as a CAD software.

Furthermore, reverse engineering was used to increase the accuracy of the CAD model. This reverse engineering involved the measurement of a clay model made as an advance conceptualization of the product, with data regarding product form characterizing this actual object used as a basis for creating CAD data. In this research, the 3D picture measurement software, “QM-3000” produced by TOPCON Techno House Corporation was used. By non-contact measurement, it was possible to convent in 3D the delicate curved line of the design, which cannot be expressed by dimension.

\section{Constructing a Method to Support the Generation of Automotive Design Ideas}

As mentioned above, it is an important research task to evaluate the "roundness" and "angle" of an automobile form, which have remained ambiguous until now, and to relate these quantities to customer sensitivity in our primary theme, development of an automobile design conception support method. Thus, establishment of the method to support the generation of automotive design ideas shown in Figure 1 for business solution is undertaken. 


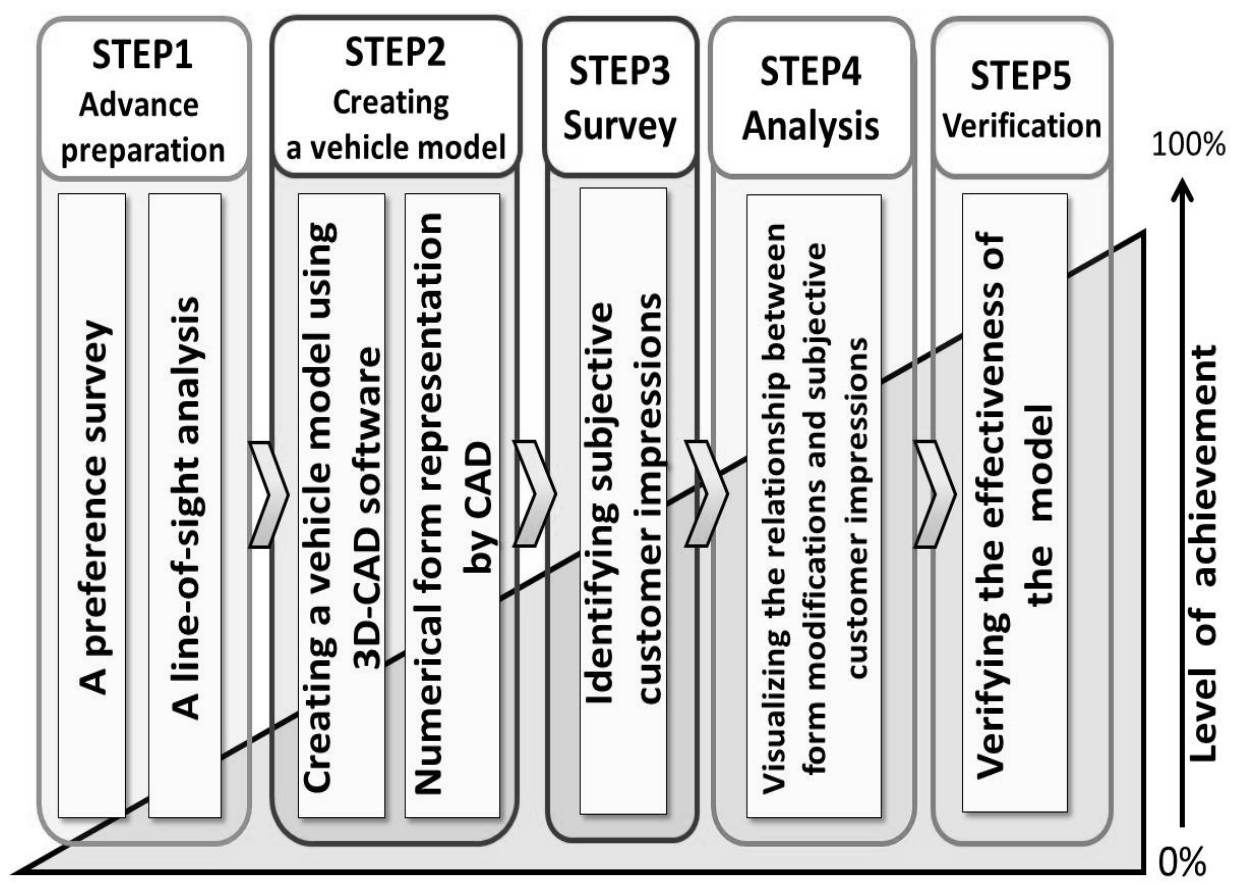

Figure 1. The method to support the generation of automotive design ideas.

\section{Using a Line-of-Sight Analysis to Determine Key Elements of Form}

A camera able to track human eye movements was used to do a line-of-sight analysis, enabling the authors to identify how people actually look at automobiles. The results indicated that most subjects observe a vehicle in a specific order: front, side, and then rear.

In terms of the relative time spent observing each part, the front: side: rear ratio was 4: 3: 3. Looking at each section individually, it was determined that viewers pay the most attention to the hood and A pillar in the front, the side window on the side, and the back window in the rear. Viewers also stopped to observe vehicles from the diagonal, with these angles making up the bulk (70\%) of total viewing time. The line-of-sight analysis made it possible to collect data in line with the way people look at actual vehicles.

\section{Creating a Vehicle Model Using 3D-CAD Software}

The process used to establish a relationship between subjective customer impressions (words) and form involves using CAD software to create several vehicle models with intentional parameters and then using them to conduct a subjective impression evaluation. During this process, it is essential to take into consideration how each basic shape might be modified. The authors used the results of the line-of-sight analysis to identify which areas of the car designers were placing the highest priority on in order to give the vehicle character.

The areas in the front that were identified as key factors were the depth of the groove lines on the edge of the hood, the swell of the hood itself, and the depth of draw in the fenders. Key factors in the back were the angle of the A pillar, the angle of the beltline, and the strength of the character line. Key factors in the rear were the angle of the $\mathrm{C}$ pillar, the width of the bumper, and the angle of the rear window. The degree to which the shapes of these elements were modified was used as a standard of comparison in the design process (see Figure 2). 

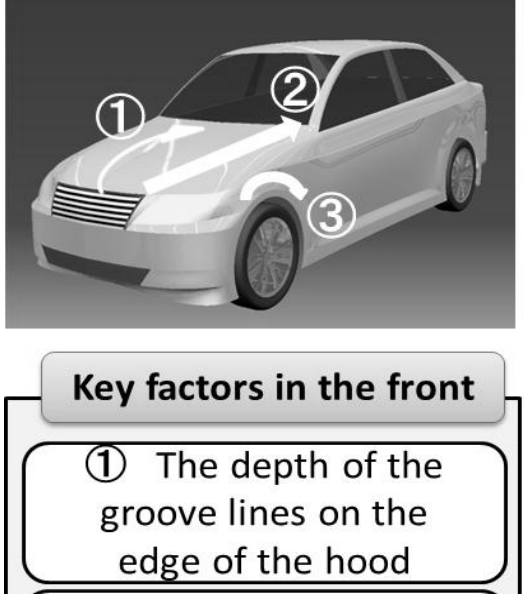

(2) The swell of the hood itself

(3) The depth of draw in the fenders

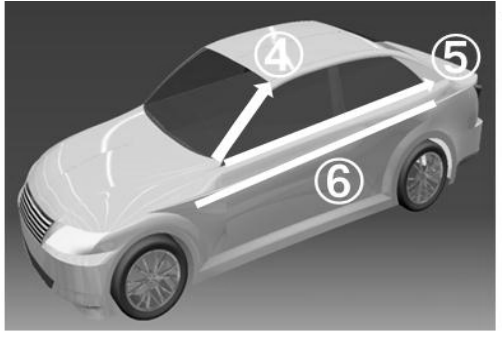

Key factors in the back

(4) The angle of the $A$ pillar

(5) The angle of the beltline

(6) The strength of the character line

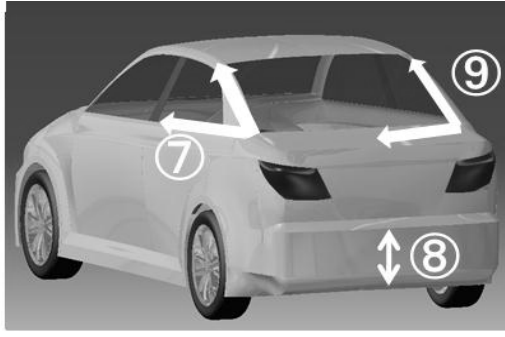

Key factors in the rear

(7) The angle of the C pillar

(8) The width of the bumper

(9) The angle of the rear window

Figure 2. Identifying key factors affecting the form of the front, side, and rear.

In this study, vehicle form was intentionally modified to see how these changes affected the impression that the vehicle made on customers. Nine different models were developed for the study, focusing on the three primary areas: side, front, and rear.
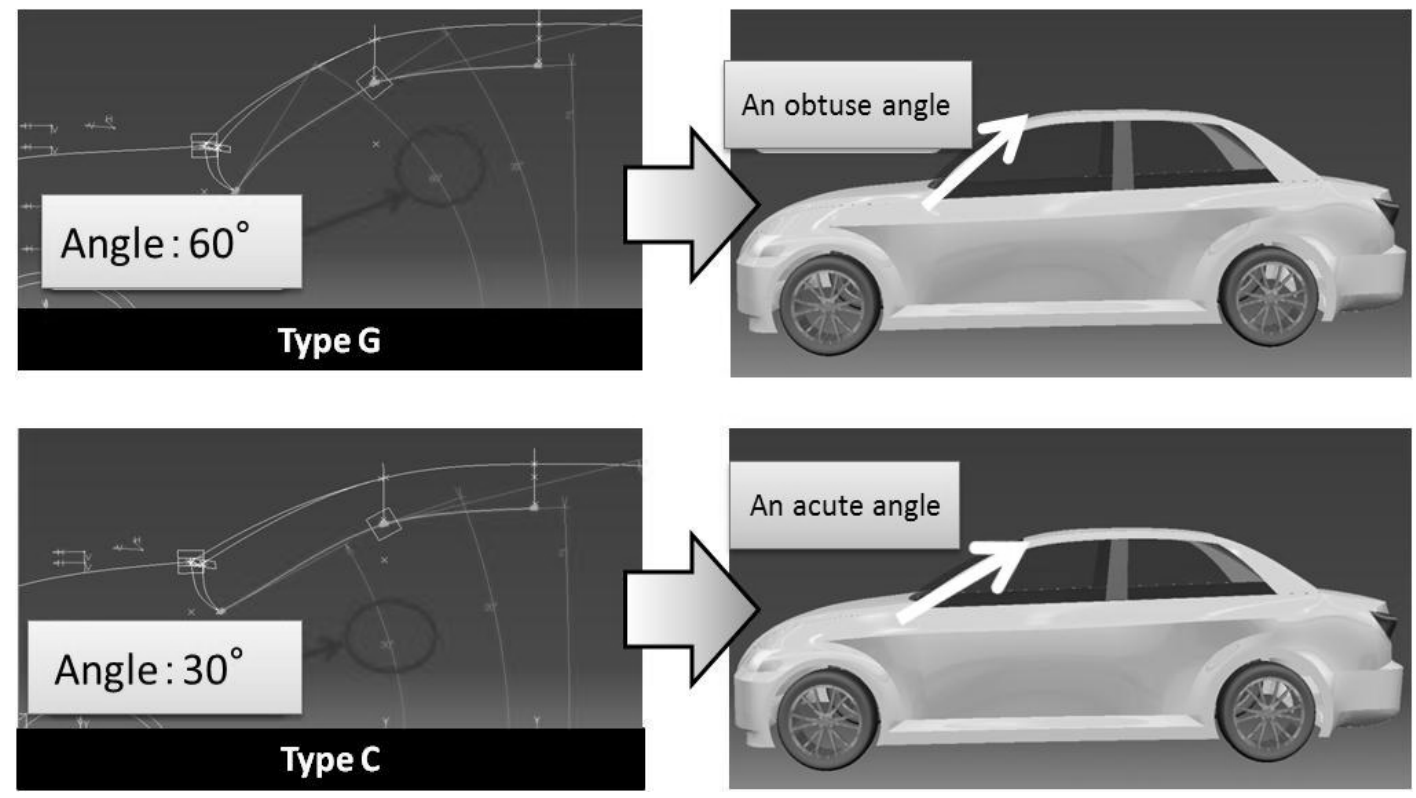

Figure 3. Examples of form modifications (side).

\section{Identifying Subjective Customer Impressions}

A customer sensibility survey was conducted to find out how each of the nine models (whose front, side, 
and rear forms had been modified) affected viewers differently. A film was produced to collect data using line-of-sight analysis, and participants were asked to answer identical survey questions for each model. The use of video allowed us to recreate a situation similar to looking at actual vehicles. The resulting data were analyzed, and relationships between form and subjective customer impressions were established. The survey targeted young adults.

In the survey, a seven-step evaluation using sensitivity concepts is performed. In order to select suitable sensitivity concepts, a bibliographic search and an investigation hearing with the designer were conducted, and 42 sensitivity words related automobile appearance were extracted. This set of concepts was then narrowed to those having a close relation, yielding the 12 sensitivity concepts as shown in Figure 4 (Asami, Owada, Murata, Takebuchi, \& Amasaka, 2010; Takimoto, Ando, Yamaji, \& Amasaka, 2010; Yazaki, Tanitsu, Hayashi, \& Amasaka, 2012).

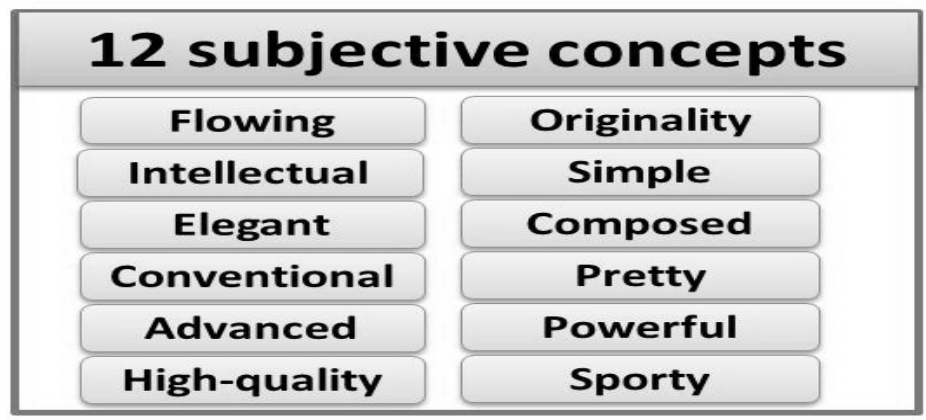

Figure 4. Selected subjective concepts.

\section{Visualizing the Relationship Between Form Modifications and Subjective Customer Impressions}

In order to identify the relative importance of subjective concepts of each shape parameter, a multiple regression analysis was conducted with subjective concepts as objective variables and shape parameters as explanatory variables. The objective variables were then weighted using each standard partial regression coefficient for the explanatory variables. The study had 12 subjective concepts, so the explanatory variables remained fixed and a multiple regression analysis was conducted for each objective variable.

The standard partial regression coefficients for the preferred subjective concepts were used to create a weighted path diagram indicating the relationships between shape parameters and subjective concepts. The diagram makes it possible to numerically identify what kind of subjective characteristics a certain model has during the trial stage. The model is also designed to help designers determine which shape parameters need to be modified in order to bring the model closer to having the desired subjective characteristics.

As an example, Figure 5 shows a weighted path diagram for shape parameters using the standard partial regression coefficient for "graceful". Looking at the diagram, we can see that the angle of the A pillar has the most powerful effect on this subjective assessment, and the fact that it is a negative value indicates that the steeper the angle of the pillar, the more "graceful" an impression a vehicle makes. If we conduct an analysis of other subjective concepts at the same time, we can visualize the relative impact of this change on each key concept.

If we want to know what effects a given shape parameter modification has on various subjective concepts, 
we can create a weighted path diagram for each concept based on that parameter. This allows us to visualize how customer impressions change as vehicle form is modified.

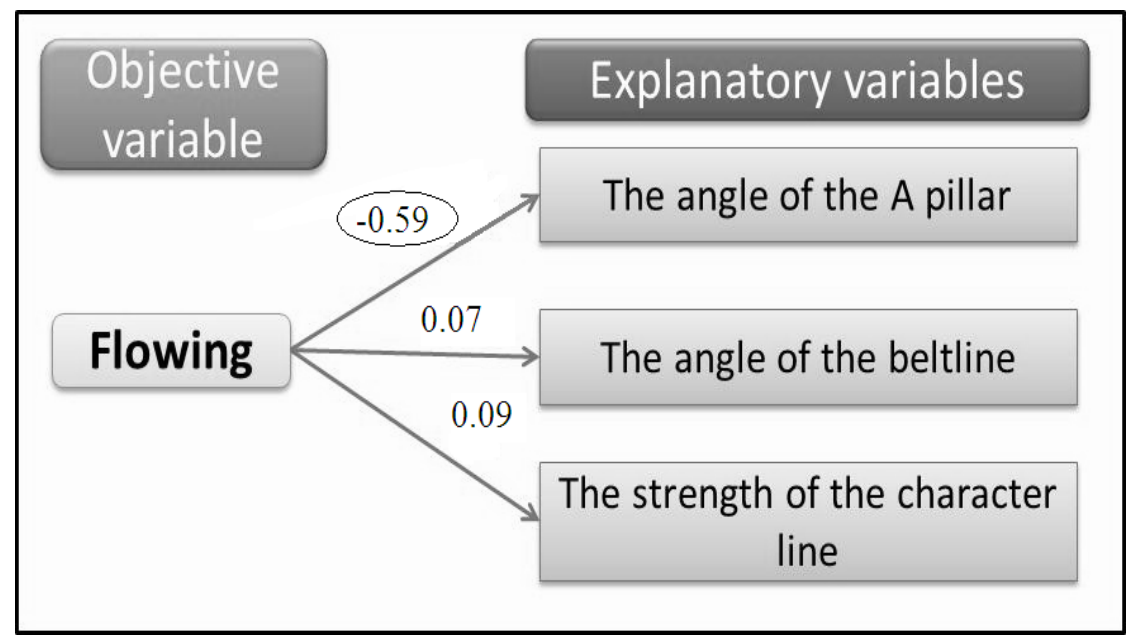

Figure 5. Sample weighted path diagram for various shape parameters (side).

\section{Verification}

Steps were taken to verify whether the form modifications and relative impact on subjective consumer impressions identified in the study were of practical use.

As an example, this study created a shape that expressed the concept of "advanced", which tends to be a favorable one among younger buyers, and used it as a model for verification. The study found eight characteristics that generate an "advanced" image: deep edges around the hood, a swell in the hood, a sharply angled A pillar, a forward-leaning beltline, an emphasized character line, a sharply angled C pillar, a wide rear bumper, and a sharply angled rear window.

These were then used as parameters to create an actual CAD design (see Figure 6). The new model was then used to conduct another verification survey, which indicated that the form of the model did in fact give younger customers the impression of being "advanced". This proved the effectiveness of the analysis results generated in this study.

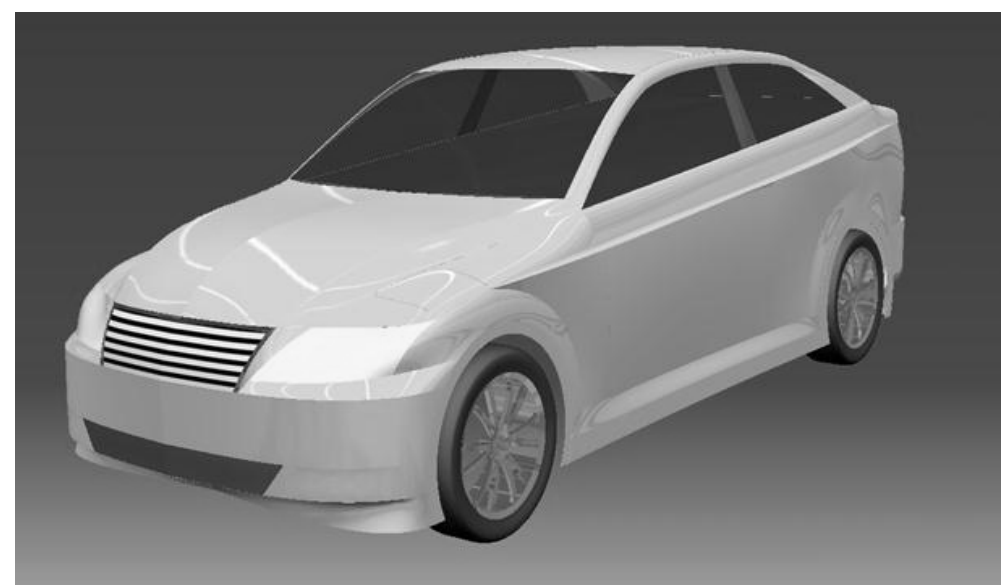

Figure 6. Completed verification model. 


\section{Conclusions}

This study looks at research on automotive form, taking previously vague concepts like "roundness" and "angularity" and quantifying them so that their relationship to subjective customer impressions can be measured.

To identify the impact of shape parameters on subjective concepts, a multiple regression analysis was conducted in order to assign a weight to each shape parameter indicating how it affected those subjective concepts. By using individual standard partial regression coefficients for explanatory variables as weights for objective variables, we were able to create a weighted path diagram outlining shape parameter-subjective concept relationships.

The diagram makes it possible to numerically identify what kind of subjective characteristics a certain model has during the trial stage, as well as determine which shape parameters need to be modified in order to bring a model closer to having the desired subjective characteristics. This has allowed us to create an approach model that supports the generation of automotive design ideas in vehicle form. When this model was applied to create a vehicle design, customers reported that it gave them the desired impression—demonstrating that the model is in fact effective.

\section{References}

Amasaka, K. (2005). Constructing a customer science application system “CS-CIANS”-Development of a global strategic vehicle "Lexus” utilizing new JIT. WSEAS Transactions on Business and Economics, 3(2), 135-142.

Amasaka, K. (2007). The validity of "TDS-DTM”: A strategic methodology of merchandise development of new JIT—Key to the excellence design “LEXUS”. International Business \& Economics Research Journal, 6(11), 105-116.

Amasaka, K., Nagaya, A., \& Shibata, W. (1999). Studies on design SQC with the application of science SQC-Improving of business process method for automotive profile design. Japanese Journal of Sensory Evaluations, 3(1), 21-29.

Aoyama, H. (2004). Digital style design based on "Kansei”. Journal of the Japanese Society for Quality Control, 34(3), 52-57.

Asami, H., Ando, T., Yamaji, M., \& Amasaka, K. (2010). A study on automobile form design support method “AFD-SM”. Journal of Business \& Economics Research, 8(11), 13-19.

Asami, H., Owada, H., Murata, Y., Takebuchi, S., \& Amasaka, K. (2010). The A-VEDAM model for approaching vehicle exterior design: Analyzing customer design preferences with CAD. Proceedings from the 9th WSEAS International Conference on System Science and Simulation in Engineering (pp. 283-288).

Li, Y., Kamaike, M., \& Ueda, E. (2007). Grasp the causal relations between design elements of passenger car and construction of needs: Analysis of the customers' activities while purchasing passenger cars in China. Bulletin of Japanese Society for Science of Design, 54(3), 65-74.

Masuyama, E. (1996). Measuring an image to appear on a heart. Industry and Science Systems.

Shinohara, A., Sakamoto, H., \& Shimizu, Y. (1996). Invitation to Kansei engineering, Morikita Shuppan.

Takimoto, H., Ando, T., Yamaji, M., \& Amasaka, K. (2010). The proposal and validity of the customer science dual system-The key to corporate management innovation. China-USA Business Review, 9(3), 29-38.

Yamaji, M., \& Amasaka, K. (2009). Intelligence design concept method utilizing customer science. The Open Industrial and Manufacturing Engineering Journal, 2, 21-25.

Yanagisawa, H., \& Fukuda, S. (2004). Development of interactive industrial design support system considering customer's evaluation: Shape design of eyeglass frame. JSME International Journal Series C: Dynamics Control Robotics Design \& Manufacturing, 47(2), 762-769.

Yazaki, K., Tanitsu, H., Hayashi, H., \& Amasaka, K. (2012). A model for design auto instrumentation to appeal to young male customers. Journal of Business Case Studies, 8(4), 417-426. 